\title{
Master's Degree
}

National Cancer Institute

\section{Source}

National Cancer Institute. Master's Degree. NCI Thesaurus. Code C39453.

The Master's Degree is an award that requires the successful completion of a program of study of at least the full-time equivalent of 1 but not more than two academic years of work beyond the bachelor's degree. 